\title{
90 degrees two-angle bending method with due to blank's elastic spring back properties
}

\author{
Elena Nesterenko ${ }^{1, *}$, and Alexander Kuzin ${ }^{1}$ \\ ${ }^{1}$ Samara University, Metal Pressure Working, Samara National Research University, 443086, \\ Moskovskoye shosse, 34, Samara, Russia
}

\begin{abstract}
Current article presents 90 degrees two-angle bending new process chart with usage of tool with elastic bars. Tool design allows considering elastic blank's springback to get more precise bending radius. Usage of elastic bars makes two-angle bending process more producible and costless as there no need to make additional operations of geometry defects correction. In that article, bending technological parameters are determined. Strain-stress blank's conditions during processing is describe. Analytical calculations are made and elastic bar sizes for different blank material are demonstrated.
\end{abstract}

\section{Introduction}

Two-angle sheet metal bending is a result of plastoelastic deformation. Deformation process carries out in different way on each side [1]. In stripe bending process, we face with generatrix curving changing on one or several segments.

Main issue to receive bended part is blank's material elastic spring back effect. After bending, a part has retained stress: extended level tries to tighten and tightened levels - to extend [2]. Because of elastic deformations during bending process, material springsback. We face with part's sizes changing in comparison with given by tool. Springback angle size depends of material elastic properties, thickness, value of deformation, angle and bending method [3]. It is difficult to consider all parameters and as a result, to get proper part we need to use additional operation such as correction, blank pre-heating, using some extra conditions during bending (distance between matrix and punch), etc.[3].

In the article we present a tool with elastic bar design that allows to make 90 degrees two-angle bending process more producible and costless [4]. Figure 1 demonstrates process scheme of 90 degrees two-angle bending process with elastic bars. All time during process bars tighten blank to punch, and as a result, that compensates springback effect (figure 1).

\footnotetext{
* Corresponding author: nesterenko77@mail.ru
} 


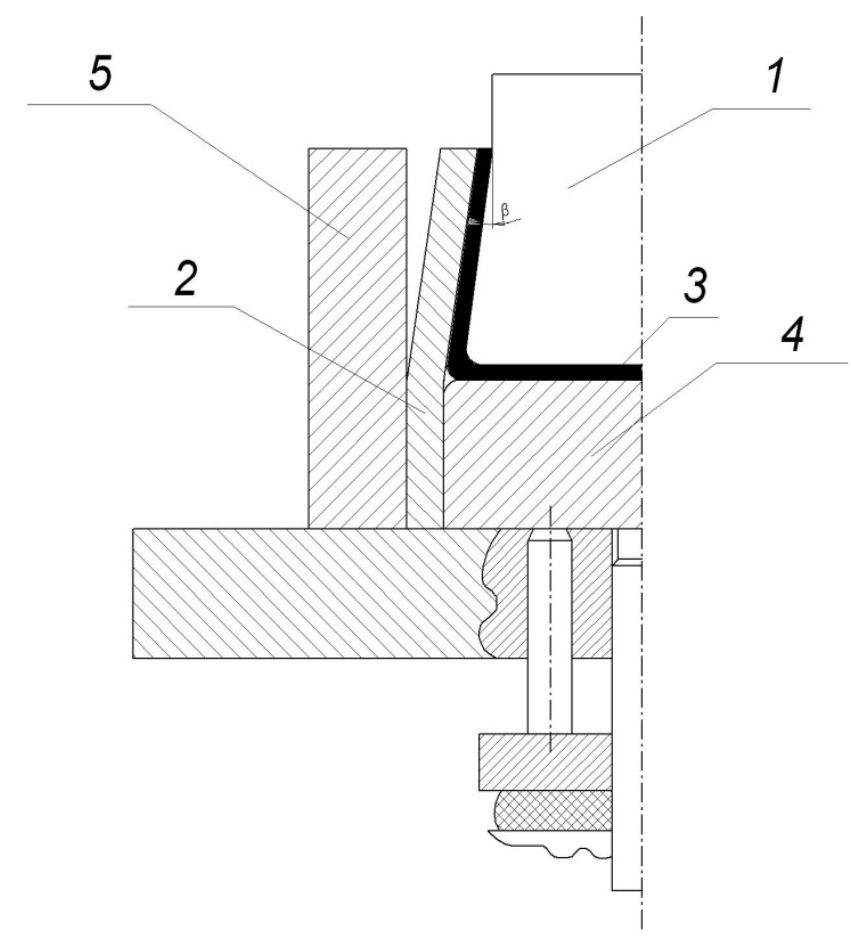

1 - punch; 2 - elastic bar; 3 - blank; 4 - ejector with buffer; 5 - matrix; $\beta$ - springback angles

Fig. 1. Process scheme of 90 degrees two-angle bending process with elastic bars.

\section{Calculation procedure}

To receive quality result it is necessary to make very resize calculations of tool parts: punch sizes, elastic bars thickness, banding angle of elastic bars before deformation. All that parameters has direct connection to material type (material's mechanical properties) and part's sizes (bending angels, bending radius, thickness).

Punch sizes are determined according to part sizes, tacking in to consideration springback angles. We calculate them according to formula:

$$
\operatorname{tg} \beta=0.75 \frac{l}{k S} \cdot \frac{\sigma_{m}}{E},
$$

here $l$-distance between matrix grips, mm; $k$ - coefficient, determine place of middle level; $S$ - blank thickness, mm; $\sigma_{m}$ - material's yield stress point, $\mathrm{MPa} ; E$ - material's elasticity modulus, MPa.

Punch angle $\alpha$ must be decreased on calculated springback angle $\beta$ :

$$
\alpha=90^{\circ}-\beta .
$$

Radius of punch is equal to radius of the part.

Dimensions of elastic bar depend on size and material of the part. Width of elastic bar $b_{1}$ is equal to width of punch $b$. Thickness of elastic bar $S_{l}$ and initial bending angle depend on thickness $S$ and part's material type. Herewith, elastic bars should not be a target of plastic yield during the whole bending process.

To determine 0 
thickness of elastic bar and initial bending angle we can use outloading theorem of Iljushin A.A. [5]. Herewith deformations of part's spot in determine moment of outloading should be equal to difference between their value at the starting moment of deformation process and elastic deformations that should take place in not stressed part under influence of external forces that are equal to differences between loads before and after outload [5]:

$$
\varepsilon_{o}=\varepsilon_{\mathrm{H}}-\varepsilon_{\mathrm{p}},
$$

here $\varepsilon_{o}$ - permanent strain; $\varepsilon_{H}-$ deformation under stress; $\varepsilon_{p}-$ deformation after outload.

In such a manner, in the beginning of bending process elastic bar that is under stress should have deformation $\varepsilon_{H}$ that is equal or higher then expression [6]:

$$
\varepsilon_{\mathrm{H}} 3 \geq \varepsilon_{\mathrm{o}}+\varepsilon_{\mathrm{p}}
$$

Deformations $\varepsilon_{o}$ and $\varepsilon_{p}$ are determine from elastic bar's mechanical properties. If we know deformation $\varepsilon_{H}$ we can determine initial bending angle of elastic bar.

At figure 2 we present bending scheme of elastic bar [7]. We can determine thickness of elastic bar.

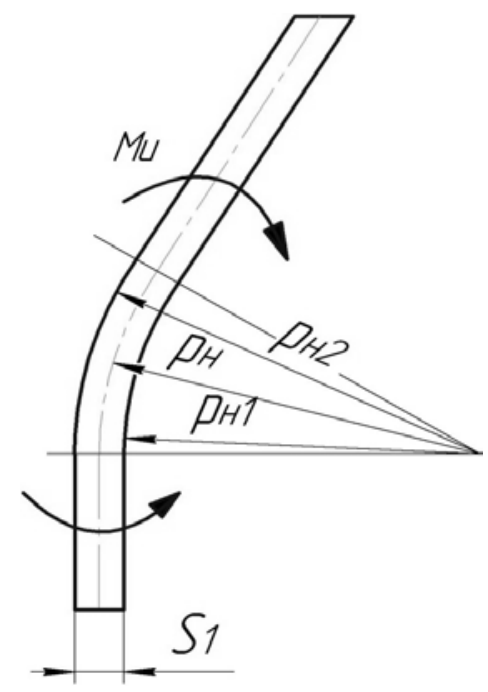

Fig.2. Elastic bar calculated bending scheme.

Final curve of middle level of elastic bar is calculated as difference between curving under stress and curving without stress [8]:

$$
\frac{1}{\rho_{\mathrm{H} 2}}=\frac{1}{\rho_{\mathrm{H} 1}}-\frac{\mathrm{M}_{\mathrm{H}}}{E \cdot I}
$$

here $\rho_{H 1}$ - internal bending radius of elastic bar, mm; $\rho_{H 2}-$ external bending radius of elastic bar, $\mathrm{mm} ; M_{u}$ - elastic deformations torque during bending, $\mathrm{H} \cdot \mathrm{mm} ; E$ - elastic bar's material elastic modulus, $\mathrm{MPa} ; \mathrm{I}$ - rectangular strip cut's mass moment of inertia relating to middle level's surface moment of inertia of the cross-sectional area of a rectangular strip with respect to a neutral surface, $\mathrm{mm}^{4}$.

$$
I=\frac{l_{1} \cdot S_{1}^{3}}{12}
$$


here $l_{l}$ - unit length, MM.

Bending torque for two-angle bending scheme is:

$$
M_{u}=\sigma_{B} \cdot \frac{S_{1}^{2} \cdot l_{1}}{4}
$$

here $\sigma_{b}$ - blank's material stress limit, MPa.

When bar is in vertical position $\rho_{\mathrm{H} 2}=\infty$, so, using formulas (5), (6) and (7) we get the following:

$$
\rho_{H 1}=\frac{E \cdot S_{1}}{3 \cdot \sigma_{B}}
$$

From formulas (5), (6), (7) and (8) for different thicknesses and width of elastic bar we get dependence:

$$
S_{1}=\frac{3 \cdot \rho_{\mathrm{H} 1} \cdot \sigma_{\mathrm{B}}}{\mathrm{E}}
$$

here $\rho_{\mathrm{H} 1}=0.5 S\left(\frac{1}{\varepsilon_{\mathrm{H}}}-1\right)[1], \mathrm{mm}, \varepsilon_{\mathrm{H}}=\frac{\sigma_{\mathrm{T}}}{\mathrm{E}_{1}} ; \sigma_{T}$ - blank's material proof stress, MPa; $E_{l}$ - blank's material elastic modulus, MPa.

\section{Results and discussions}

In table 1 we demonstrate values of elastic bar's thickness for different materials. Elastic bars are made of steel $1060\left(\sigma_{6}=980 \mathrm{M \Pi а}, \mathrm{E}=2.04 \cdot 10^{5} \mathrm{M \Pi а}\right)$.

Table 1. Values of elastic bar's thickness for different materials and blank's thickness $S=1 \mathrm{~mm}$.

\begin{tabular}{|c|c|c|c|c|}
\hline Material & $E_{1}, 10^{5} M P a$ & $\begin{array}{c}\sigma_{T}, \\
M \Pi a\end{array}$ & $\varepsilon_{H}$ & $S_{1, m m}$ \\
\hline $\mathrm{C} 27200$ & 1.30 & 300 & 0.0042 & 1.7 \\
\hline 2124 & 0.71 & 250 & 0.0035 & 2 \\
\hline $1050 \mathrm{~A}$ & 0.69 & 145 & 0.0021 & 3.4 \\
\hline $\mathrm{A} 107$ & 2.00 & 400 & 0.0020 & 3.6 \\
\hline $\mathrm{A} 622$ & 2.07 & 330 & 0.0015 & 4.5 \\
\hline
\end{tabular}

Analyzing the results, we can conclude that the thickness of the elastic bars depends on the ratio of the proof stress and the modulus of elastic of the blank's material. The smaller the ratio, the greater the thickness of the elastic bars.

\section{Conclusion}

In that way, we determine necessary technological and geometrical parameters that allow choosing optimal thickness of elastic bar. During process designing, we calculate stresses and deformations that demonstrate no elastic deformation of bars. Designed stamping scheme with use of elastic bars demonstrates good perspectives in technology and is recommended for use in real manufacturing. 


\section{References}

1. V.P. Romanovsky, Handbook of cold stamping (Moscow, Engineering, 1979).

2. M.V. Storogev, Theory of metal forming (Moscow, Engineering, 1977).

3. Z. Marciniak, J. L., Duncan and S.S. Hu, Mechanics of Sheet Metal Forming, (2002).

4. Die for bending flat parts with elastic bar, pat. 153887 RU: MПК B21D5/02/, I.P. Popov, E.S. Nesterenko, A.O. Kuzin, (2015).

5. Schuler GmbH., Metal forming, (Handbook, Springer, 2012).

6. A.A. Iljushin, Plasticity, (Moscow, Leningrad OGIZ, 1948).

7. I.P. Popov, E.S. Nesterenko, A.O. Kuzin, Decrease of elastic stringiness during twoangle bending in die with elastic plate, Moscow, Engineering, v. 5(13), pp.121-124. (2013).

8. I.P. Popov, E.S. Nesterenko, A.O. Kuzin, Probe of strains and the voltages arising at two-angle bending in a die with elastic plate, Izvestia SNC RAN, v. 4(18), pp. 25-30 (2016). 